\title{
Candida-associated denture stomatitis
}

\author{
Carmen Salerno ${ }^{1}$, Michelangelo Pascale ${ }^{1}$, María Contaldo ${ }^{1}$, Vincenzo Esposito ${ }^{1}$, Maurizio Busciolano ${ }^{1}$, \\ Lucio Milillo $^{3}$, Agostino Guida ${ }^{1}$, Massimo Petruzzi ${ }^{4}$, Rosario Serpico ${ }^{2}$
}

${ }^{1}$ SUN-Dip. di Discipline Odontostomatologiche, Ortodontiche e Chirurgiche 0815665476

${ }^{2}$ Dipartimento assistenziale di odontoiatria: programma di attività di patologia delle mucose orali. Responsabile: Prof. R. Serpico

${ }^{3}$ Dipartimento di Scienze Chirurgiche, Facoltà di Medicina e Chirurgia, Università degli Studi di Foggia, Foggia, Italy

${ }^{4}$ Dipartimento di odontostomatologia e chirurgia, Università di Bari

Correspondence:

Via De Crecchio, 6

80138 NAPOLI

carmensalerno1@virgilio.it

Received: 24/02/2010

Accepted: $27 / 06 / 2010$

\author{
Salerno C, Pascale M, Contaldo M, Esposito V, Busciolano M, Milillo L, \\ Guida A, Petruzzi M, Serpico R. Candida-associated denture stomatitis. \\ Med Oral Patol Oral Cir Bucal. 2011 Mar 1;16 (1):e139-43. \\ http://www.medicinaoral.com/medoralfree 01/v16i2/medoralv16i2p139.pdf \\ Article Number: $16864 \quad$ http://www.medicinaoral.com/ \\ (C) Medicina Oral S. L. C.I.F. B 96689336 - pISSN 1698-4447 - eISSN: 1698-6946 \\ eMail: medicina@medicinaoral.com \\ Indexed in: \\ Science Citation Index Expanded \\ Journal Citation Reports \\ Index Medicus, MEDLINE, PubMed \\ Scopus, Embase and Emcare \\ Indice Médico Español
}

\begin{abstract}
Candida albicans is a dimorphic yeast strongly gram positive able to live as normal commensal organism in the oral cavity of healthy people. It is the yeast more frequently isolated in the oral cavity. Under local and systemic factors related to the host conditions, it becomes virulent and responsible of oral diseases known as oral candidiasis. It has been shown that the presence of denture is a predisposing factor to the onset of pathologies related to C. albicans. Clinical studies have shown that $\mathrm{C}$. albicans is not only able to adhere to the mucous surfaces, but also to stick to the acrylic resins of the dental prostheses. Both the plaque accumulated on the denture and the poor oral hygiene contribute to the virulence of Candida, offering the clinical picture of Candida-associated denture stomatitis. The therapeutic strategies currently adopted in the clinical practice to overcome these fungal infections provide for the use of topical and/or systemic antifungal and topical antiseptics and disinfectants, the irradiation with microwaves and the accurate mechanical removal of the bacterial plaque from the denture surfaces and from the underlying mucosa. A correct oral hygiene is important for the control of the bacterial biofilm present on the denture and on the oral mucosa and it is the fundamental base for the prophylaxis and the therapy of the Candidaassociated denture stomatitis.
\end{abstract}

Key words: Candida albicans, denture stomatitis, dental plaque, antifungal drugs.

\section{Introduction}

Candida albicans is an innocuous commensal of the microbial communities of the human oral cavity. Its primary location is the posterior tongue and other oral sites as the mucosa, while the film that covers the dental surfaces is colonized secondarily (1). Frequently, when the host defense system suffers because of any alterations, like immunodeficiency, C. albicans become virulent and generates candidiasis, that can be manifested through various clinical forms, involving one or more oral sites, up to affect the whole oral cavity and to disseminate into invasive forms. Candida-associated denture stomatitis is a very common inflammatory process affecting about $60 \%$ of the subjects carrier of a prosthesis (2). 


\section{Epidemiology}

Candida-associated denture stomatitis has been found in $60-65 \%$ of the subjects carriers of prosthesis (3) with more diffused clinical manifestations, but considering also the subjects that do not manifest clinical signs of inflammation, the percentage increases to $75 \%$ of the population bearer of prosthesis. C. albicans has been shown to be the principal Candida strain responsible for inflammatory pathology (4) even if various studies have isolated other species of Candida involved in the patogenesis of the oral candidiasis, as C. dubliniensis, C. parapsilosis, C. krusei, C. tropicalis and, above all, C. glabrata (5). However, C. albicans remains the principal pathogen, due to its capability to stick and proliferate through the hard and soft tissues of the oral cavity and to produce a complex and heterogeneous bacterial biofilm.

\section{Pathogenesis}

The pathogenesis of the Candida-associated denture stomatitis is elaborate and multifactorial. It includes local and systemic factors related to the host and to the Candida capability to adhere and proliferate in the host epithelial tissues (6). Candida-associated denture stomatitis is able to rise up when the conditions of the micro oral environment are favourable for the growth and the adhesion of the yeast and also when systemic factors of the host bring to a depression of the mechanisms of defence.

-Systemics factors

Diabetes

The saliva of diabetics favours the growth of C. albicans in vitro and it has been shown that on the denture surfaces of diabetic there are more elevated counts of colonies of the yeast by comparison with the non diabetic subjects (7).

Deficiency of nutritional factors

Some authors report the sideropenic anaemia and high levels of cholesterol as causes of candidiasis (8).

\section{Kidneys affections}

These affections are frequent in individuals of advanced age. The repeated treatments with antibiotics and sulphonamides can be predisposing factors because of the microbial alterartions that they provoke in the oral cavity (9).

\section{Xerostomy}

Qualitative and quantitative alterations of the salivary flow in elderly patients is probably secondary to the assumption of drugs, above all the antihypertensive ones, rather than a primary functional deficit. Such reduction has been shown to act as predisposing factor to the virulence of the C. species (10).

- Local factors

Traumas

Nyquist considered traumas as the main liable to determine Candida-associated denture stomatitis with none association with the microbial communities and the

Table 1. Evolution of the Candida infection according to the time of amorolfine application

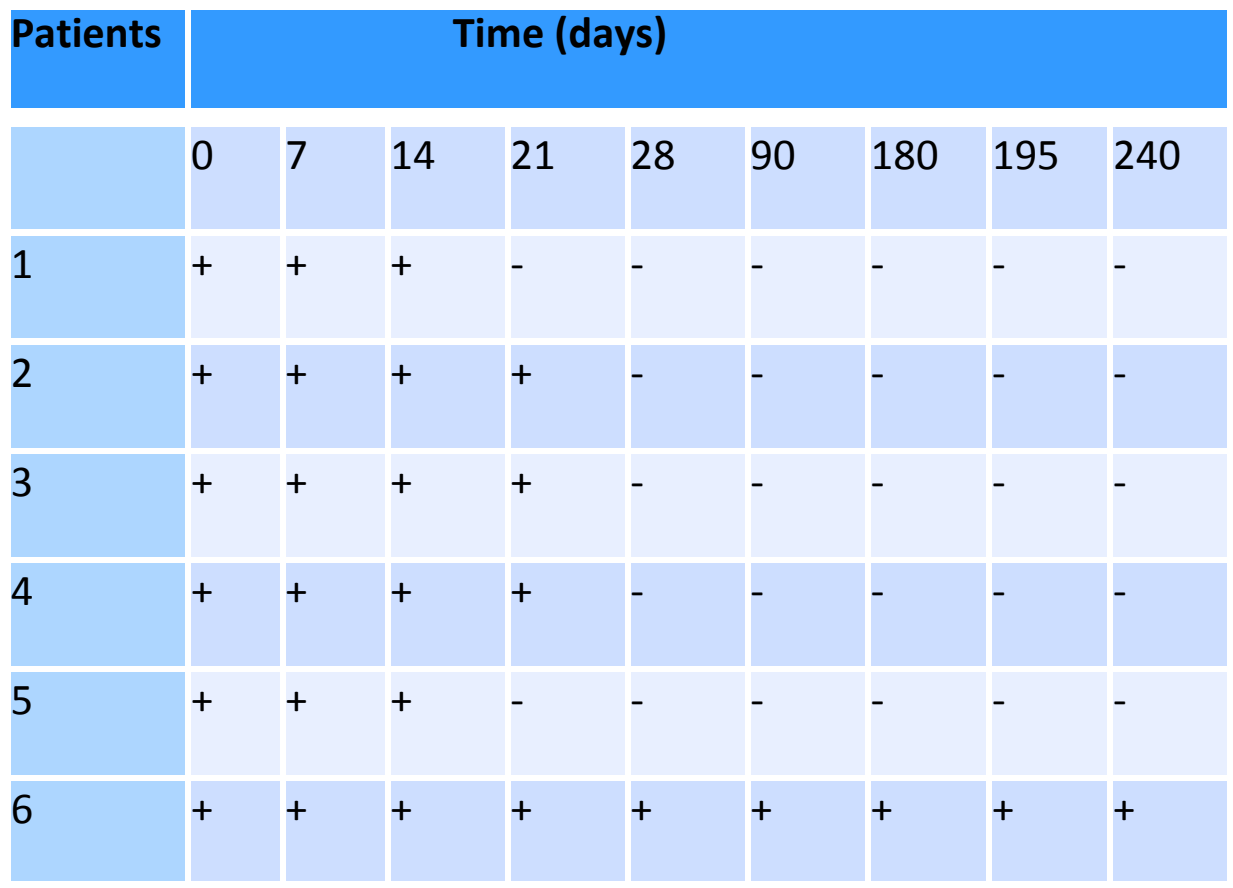


presence of denture (11). Subsequently, Cawson showed that traumas and Candida infection are together responsible for the pathogenesis of the denture stomatitis. The latest study pointed out that trauma alone does not induce pictures of generalized denture stomatitis but, rather, it could be the cause of localized forms. Instead, in the generalized forms the principal pathogenetic role is played by Candida albicans. In this case, trauma could act as co-factor that favours the adhesion and the penetration of the yeast, sustains phlogosis of the palate and increases the permeability of the epithelium to toxins and soluble agents produced by Candida yeast. Denture trauma due to unstable dentures is also one of etiological factors of denture stomatitis. Immunohistochemical analysis of the mucosal tissue involved in denture stomatitis has demonstrated a possible role of trauma in the variation of expression of the basement membrane antigens (11).

The saliva

The role of the saliva in the colonization of $\mathrm{C}$. albicans is still controversial. Some studies have shown that it reduces the adhesion of $\mathrm{C}$. albicans. In fact, the saliva possesses defensive molecules as lysozyme, lactoferrine, calprotectin, IgA that decrease the adhesion of Candida to the oral surfaces (12). In other studies, it has been shown that salivary proteins as the mucines and the statherins may act as adhesion receptors used by the mannoproteins present in the Candida species (1). The decrease or the complete absence of saliva in individuals with xerostomy induces the change and the imbalance of the normal microbial communities favouring the proliferation of bacteria as Staphylococcus aureus, that inhibits the normal adaptation of the commensals (1). Besides, the presence of a low level of $\mathrm{pH}$ and of a high oxygen tension reduces the growth of some commensals while increases the proliferation of C. species, Streptococcus mutans and Lactobacillus. Recent study show the saliva plays a dual role in the adhesion of $\mathrm{C}$. albicans o plastic material used to make a dental prosthesis, decresing adhesion of germinated cell an enhancing the adhesion of yeast cell (13).

$\mathrm{pH}$ of the oral cavity

Low levels of $\mathrm{pH}$ can favour the adhesion and the proliferation of Candida yeast. In fact, a $\mathrm{pH}$ equal to 3 is optimal not only for the adhesion of the yeasts, but also for the enzymatic activity of the proteinases that, together with the lipases, are the most important factors of virulence of Candida because of their cytotoxic and cytolytic effects. Moreover, high levels of carbohydrates present in the saliva can act as an additional nourishing source for the Candida yeasts, that, by metabolizing these sugars, produce acid metabolic products and contribute to maintain low the environmental $\mathrm{pH}$ (1).

Permeability of the acrylic resins

Initially, the adhesion of Candida depends on the mi- croporosity present on the surface of the denture (5). Such irregularities of surface make possible the yeasts to nest and make difficult to eliminate bacteria by mechanics and chemical hygiene manoeuvres; therefore, in presence of poor oral hygiene, Candida can penetrate, stick and aggregate with the bacterial communities, as Streptococcus sanguis, Streptococcus gordonii, Streptococcus oralis and Streptococcus anginosus (S. milleri) by the interactions between proteins and carbohydrates (1).

\section{Presence of microbial plaque}

Various microbiologic studies underlined that the plaque accumulated on the dentures during stomatitis has a complex composition, represented above all by Gram-positive bacteria (1), as Streptococcus sanguis, S. gordonii, S.oralis, S. anginosus, Staphylococci and rods as Actinomycetes predominatly, followed by Lactobacillus. The microorganisms present in the oral cavity interact between them in various ways, as using directly their own metabolic products or exchanging themselves molecular signals. Several studies have shown that the coaggregation includes protein-carbohydrates interactions . Candida has been shown to be the predominant pathogen. First of all, it has been seen that patients with denture stomatitis show an increase in the presence of Candida when compared to the controls. Secondly, the patients response to the antimycotic therapy with a drastic decrease of the colonies present in the denture plaque. As third point, we must recall that the mass of yeast cell is 50 times greater than that of a coccus and that the mass of an hypha can be greater hundreds of times than that one of a rod. Candida, therefore, plays a key pathogenetic role in the onset of the denture stomatitis, even if the cooperative role practiced by the bacterial plaque present on the denture must not be neglected (12).

\section{Adhesion}

The ability of Candida to pass through the tissues is the first step of the infectious process $(6,14)$. It has been observed that the forms endowed with hyphae are able to stick and to invade more quickly the tissues of the host. The complex mechanism of interaction between Candida and the host provides for the interaction between cellular ligands of Candida and cellular receptor of the host. The first ones consist in the mannoproteins present on the cellular surface. In fact the yeast produces extracellular polymeric material containing mannoproteins. The interaction with the epithelial cells happens between the mannoproteins and the fucose or the $\mathrm{N}$ acetylglucosamine present on the surface proteins of the epithelial cells (1).

-Pathogenetic theory

The presence of bacteria as Streptococci and Actinomycetes induces the organism to produce proteases as IgA1 and enzymes as amino-peptidases, hyaluronidas- 
es, chondroitinases and neuraminidases, able to degrade the oral epithelium. These harmful products, storing in close contact with the oral mucosa, determine an increase in the inflammatory exudate that not only favours the bacterial colonization, but also the yeast proliferation since Candida colonizes more easily the mucosa in contact with the denture surface respect to the rest of the buccal mucosa. The proteases can increase the pathogenic potential of the bacterial substances, leading to destroy the salivary immunoglobulines. The following immunitary system response to the plaque deposits, is responsible for the inflammatory lesions. Experimental data have shown that a delayed hypersensitivity reaction towards Candida albicans notably contributes to the inflammatory answer and that the exfoliation of the epithelial cells, leading to the epithelial atrophy, rather than the invasion of hyphae, is the typical feature of the denture stomatitis.

\section{Therapy}

The treatment of Candida-associated denture stomatitis is complex because of its multifactorial etiology (14). The therapeutic strategy still adopted includes the use of topical and systemic antifungal drugs, the use of preservatives and disinfectants, the irradiation with microwaves and the scrupulous removal and control of the plaque present on the denture and on the oral mucosa. -Antifungal drugs

The antifungal treatments more used are antifungal suspensions based on nystatin, amphotericin-B, miconazole and fluconazole. Almost all drugs generally produce a complete remission of symptoms within 12-14 days. Webb says that Epstein et al. showed the importance of antifungal therapy in the treatment and prevention of oral candidiasis (14). They noticed that nystatin and amphotericin-B, because of their binding to the ergosterol on the Candida cellular membranes, causes changes in the permeability of the cell membrane, leading to their penetration into the cells and causing finally the cell death. Tobudic et al.(15) bring that Merkel and Phelps showed that sub-lethal doses of amphotericin-B inhibits the adhesion of Candida to the cultures of mammalian cells, and that the blastospores in the stage of active growth are more sensitive to the drug. Other studies have shown that sub-inhibiting doses of nystatin, amphotericin-B and miconazole inhibit adhesion of Candida to epithelial cells (15).

Among the topically antifungal drugs used, the efficacy of "locetar" was noted (16); it is used in the treatment of onychomicosis. Amorolfine belongs to a new class of chemical antifungal. Its fungistatic and fungicide effect is based on the alteration of the fungal cell membranes, in particular at the level of the sterols biosynthesis. In this way, the content of ergosterol is reduced, and at the same time not usual planar sterols accumulate. Amo- rolfine possesses a broad spectrum of action in vitro, and it is particularly effective versus dirofilariosis (trichophyton, microsporum, epidermophyton), yeasts (Candida), moulds (Alternaria, Hendersonula); with the exception of Actinomycetes, the bacteria are not sensitive to amorolfine (Table 1).

- Preservative and disinfectant agents

The use of antiseptic substances as the $0.2 \%$ chlorhexidine gluconate administered 3 or 4 times a day, is capable of carrying on a significant reduction of the plaque but it has not a significant effect on the reduction of the colonies of Candida (14). More encouraging results are obtained when the dentures are immersing into $2 \%$ chlorhexidine as aid to topical therapy. Note that the chlorhexidine never must be administered at the same time with the nystatin since it inhibits the antifungal capacity. Another antiseptic substance used is sodium hypochlorite. It is proven that by diving the denture in a solution of $0.02 \%$ sodium hypochlorite, the number of Candida and bacteria amount on the denture surface effectively decrease. Unfortunately, sodium hypochlorite may not be used for an indeterminate period of time according to its ability to damage the prosthetic handiwork (17).

\section{- Microwave irradiation}

Irradiation with microwave has been proposed as a quick effective and cheap method for the denture disinfection. In vitro the exposure to the microwaves was able to cause the cell death of Candida albicans. Clinical assessment has proved the real effectiveness of this methodology to disinfect the denture and to treat Candida-associated denture stomatitis (18) by the exposure of the denture to the microwaves ( $350 \mathrm{Watt}, 2450 \mathrm{MHz}$ ) for 6 minutes, removing the presence of Candida and bacteria. However this treatment is responsible to produce conformational changes on the denture, according to the duration of the treatment and therefore on the possibility of adopting this method together with maneuvers of oral and denture hygiene. In fact, according to the quantum theory, the waves formation induces a production of energy that could interfere with the dimensional stability of the denture (19).

\section{-Scrupulous removal of denture plaque}

The poor oral and denture hygiene are fundamental in the onset of disease, demonstrating the importance of the cleanliness of the denture through mechanical and chemical methods (20). An efficient control of the microbial plaque on the denture remains the most important and certain procedure to follow.

A good oral hygiene can be alone effective in treating denture stomatitis as well as when it is adopted in association with systemic and topical antifungal drugs. The hygiene control of denture is also essential to avoid relapses of pathology following treatment with antifungal drugs and, therefore, it is an important measure for 
the prophylaxis of candidiasis. Both the prosthesis that oral mucosa in contact with it must be involved in procedures for oral hygiene through brushing them after each meal with water or chemical agents. The patients should also be instructed to remove the denture during night and to leave it dry; in addiction, during therapy for stomatitis, the prosthesis should be removed for at least two weeks.

\section{Conclusions}

Candida-associated denture stomatitis remains the more frequent form of oral candidiasis (25-65\% especially in carriers of denture) with preferential localization to the palatal mucosa. Among the predisposing local factors the main one is the accumulation of microbial plaque on the surface of the denture in contact with the mucosa. Candida-associated denture stomatitis, even if asymptomatic, should be treated as it may act as reservoir for infections more extensive and encourage the resorption of the alveolar bone. Both in the therapy that in prevention, the more effective treatment is the eradication and control of the microbial plaque.

\section{References}

References with links to Crossref - DOI

1. Webb BC, Thomas CJ, Willcox MD, Harty DW, Knox KW, Webb $\mathrm{BC}$, et al. Candida-associated denture stomatitis. Aetiology and management: a review. Part 1. Factors influencing distribution of Candida species in the oral cavity. Aust Dent J. 1998;43:45-50.

2. Geerts GA, Stuhlinger ME, Basson NJ. Effect of an antifungal denture liner on the saliva yeast count in patients with denture stomatitis: a pilot study. J Oral Rehabil. 2008;35:664-9.

3. Webb BC, Thomas CJ, Willcox MD, Harty DW, Knox KW. Candida-associated denture stomatitis. Aetiology and management: a review. Part 2. Oral diseases caused by Candida species. Aust Dent J. 1998;43:160-6.

4. Budtz-Jörgensen E, Stenderup A, Grabowski M. An epidemiologic study of yeasts in elderly denture wearers. Community Dent Oral Epidemiol. 1975;3:115-9.

5. Ferreira MA, Pereira-Cenci T, Rodrigues de Vasconcelos LM, Rodrigues-Garcia RC, Del Bel Cury AA. Efficacy of denture cleansers on denture liners contaminated with Candida species. Clin Oral Investig. 2009;13:237-42.

6. Bilhan H, Sulun T, Erkose G, Kurt H, Erturan Z, Kutay O, et al. The role of Candida albicans hyphae and Lactobacillus in denturerelated stomatitis. Clin Oral Investig. 2009;13:363-8.

7. Yuen HK, Wolf BJ, Bandyopadhyay D, Magruder KM, Salinas CF, London SD. Oral health knowledge and behavior among adults with diabetes. Diabetes Res Clin Pract. 2009;86:239-46.

8. Paillaud E, Merlier I, Dupeyron C, Scherman E, Poupon J, Bories $\mathrm{PN}$. Oral candidiasis and nutritional deficiencies in elderly hospitalised patients. Br J Nutr. 2004;92:861-7.

9. Golecka M, Ołdakowska-Jedynak U, Mierzwińska-Nastalska E, Adamczyk-Sosińska E. Candida-associated denture stomatitis in patients after immunosuppression therapy. Transplant Proc. 2006;38:155-6.

10. Campisi G, Panzarella V, Matranga D, Calvino F, Pizzo G, Lo Muzio L, et al. Risk factors of oral candidosis: a twofold approach of study by fuzzy logic and traditional statistic. Arch Oral Biol. 2008;53:388-97.

11. Emami E, de Grandmont P, Rompré PH, Barbeau J, Pan S, Feine JS. Favoring trauma as an etiological factor in denture stomatitis. J Dent Res. 2008;87:440-4.
12. Baena-Monroy T, Moreno-Maldonado V, Franco-Martínez F, Aldape-Barrios B, Quindós G, Sánchez-Vargas LO. Candida albicans, Staphylococcus aureus and Streptococcus mutans colonization in patients wearing dental prosthesis. Med Oral Patol Oral Cir Bucal. 2005;10 Suppl 1:E27-39.

13. Azcurra AI, Barembaum SR, Bojanich MA, Calamari SE, Aguilar J, Battellino LJ, et al. Effect of the high molecular weight chitosan and sodium alginate on Candida albicans hydrophobicity and adhesion to cells. Med Oral Patol Oral Cir Bucal. 2006;11:E120-5.

14. Webb BC, Thomas CJ, Willcox MD, Harty DW, Knox KW. Candida-associated denture stomatitis. Aetiology and management: a review. Part 3. Treatment of oral candidosis. Aust Dent J. 1998;43:244-9.

15. Tobudic S, Kratzer C, Lassnigg A, Graninger W, Presterl E. In vitro activity of antifungal combinations against Candida albicans biofilms. J Antimicrob Chemother. 2010;65:271-4.

16. Milillo L, Lo Muzio L, Carlino P, Serpico R, Coccia E, Scully C. Candida-related denture stomatitis: a pilot study of the efficacy of an amorolfine antifungal varnish. Int J Prosthodont. 2005;18:55-9.

17. Kadir T, Gümrü B, Uygun-Can B. Phospholipase activity of Candida albicans isolates from patients with denture stomatitis: the influence of chlorhexidine gluconate on phospholipase production. Arch Oral Biol. 2007;52:691-6.

18. Agha-Hosseini F. Fluconazole and/or hexetidine for management of oral candidiasis associated with denture-induced stomatitis. Oral Dis. 2006;12:434.

19. Sanitá PV, Vergani CE, Giampaolo ET, Pavarina AC, Machado AL. Growth of Candida species on complete dentures: effect of microwave disinfection. Mycoses. 2009;52:154-60.

20. Dorocka-Bobkowska B, Konopka K. Susceptibility of candida isolates from denture-related stomatitis to antifungal agents in vitro. Int J Prosthodont. 2007;20:504-6. 